\title{
Experiments at the GELINA facility for the validation of the self-indication neutron resonance densitometry technique
} \author{
Nicolas Pauly ${ }^{2}$, Peter Schillebeeckx ${ }^{3}$, and Klaas van der Meer ${ }^{1}$ \\ 1 Society and Policy Support, Belgian nuclear research centre SCK $\bullet$ CEN, Mol, Belgium \\ 2 Ecole polytechnique de Bruxelles - Université libre de Bruxelles ULB, Brussels, Belgium \\ 3 European Commission, Joint Research Centre JRC, Directorate G, Geel, Belgium
}

Riccardo Rossa ${ }^{1,2, a}$, Alessandro Borella ${ }^{1}$, Jan Heyse ${ }^{3}$, Stefan Kopecky ${ }^{3}$, Pierre-Etienne Labeau ${ }^{2}$, Carlos Paradela $^{3}$,

\begin{abstract}
Self-Indication Neutron Resonance Densitometry (SINRD) is a passive non-destructive method that is being investigated to quantify the ${ }^{239} \mathrm{Pu}$ content in a spent fuel assembly. The technique relies on the energy dependence of total cross sections for neutron induced reaction. The cross sections show resonance structures that can be used to quantify the presence of materials in objects, e.g. the total cross-section of ${ }^{239} \mathrm{Pu}$ shows a strong resonance close to $0.3 \mathrm{eV}$. This resonance will cause a reduction of the number of neutrons emitted from spent fuel when ${ }^{239} \mathrm{Pu}$ is present. Hence such a reduction can be used to quantify the amount of ${ }^{239} \mathrm{Pu}$ present in the fuel. A neutron detector with a high sensitivity to neutrons in this energy region is used to enhance the sensitivity to ${ }^{239} \mathrm{Pu}$. This principle is similar to self-indication cross section measurements. An appropriate detector can be realized by surrounding a ${ }^{239} \mathrm{Pu}$-loaded fission chamber with appropriate neutron absorbing material. In this contribution experiments performed at the GELINA time-of-flight facility of the JRC at Geel (Belgium) to validate the simulations are discussed. The results confirm that the strongest sensitivity to the target material was achieved with the self-indication technique, highlighting the importance of using a ${ }^{239} \mathrm{Pu}$ fission chamber for the SINRD measurements.
\end{abstract}

\section{Introduction}

The self-indication neutron resonance densitometry (SINRD) measures the passive neutron emission from spent fuel [1]. The SINRD technique aims at measuring the attenuation of the neutron flux around the $0.3 \mathrm{eV}$ energy region as a way to directly quantify the ${ }^{239} \mathrm{Pu}$ mass in the spent fuel.

The cross-section expresses the interaction probability between a certain nuclide and an incoming neutron, and it is specific for each nuclide. The cross-section of ${ }^{239} \mathrm{Pu}$ shows a resonance around $0.3 \mathrm{eV}$. Therefore, significant neutron absorption is expected in correspondence of the $0.3 \mathrm{eV}$ resonance due to the presence of ${ }^{239} \mathrm{Pu}$ in spent fuel.

The application of SINRD to spent fuel measurements was proposed in [2-5] by measuring a fuel assembly under water and by placing a set of ${ }^{235} \mathrm{U}$ fission chambers on one side of the assembly.

Another approach was developed recently at SCK $\bullet \mathrm{CEN}$ [6-8] by measuring in dry conditions a fuel assembly surrounded by a thick slab of polyethylene. The ${ }^{239} \mathrm{Pu}$ mass in the fuel assembly is estimated with the SINRD signature $\left(R_{S I}\right)$ defined in Formula (1).

$$
R_{S I}=\frac{C_{F}}{C_{G d}-C_{C d}}
$$

A measurement with a ${ }^{238} \mathrm{U}$ fission chamber is foreseen for the neutron counts in the fast energy region $\left(C_{F}\right)$, whereas a ${ }^{239} \mathrm{Pu}$ fission chamber covered by a thin foil of

a e-mail: rrossa@sckcen.be either $\mathrm{Gd}$ or $\mathrm{Cd}$ in the other measurements $\left(C_{G d}, C_{C d}\right)$. These elements were chosen because they show a cut-off energy for neutron absorption slightly below and above $0.3 \mathrm{eV}$, respectively. Due to this property these materials are called SINRD filters. By taking the difference of the neutron counts measured with the two filters, the neutron flux in the energy region close to the ${ }^{239} \mathrm{Pu}$ resonance is estimated. In addition, the use of a ${ }^{239} \mathrm{Pu}$ fission chamber enhances the detection of neutrons with energy close to $0.3 \mathrm{eV}$ according to the self-indication effect [9].

\section{Experimental setup}

\subsection{Transmission measurements}

Transmission measurements can be used for the determination of total neutron cross-section data [9-11], and the schematic view of the measurement setup is shown in Fig. 1. The experiments were carried out to measure the transmitted neutron flux through several samples of Gd and $\mathrm{Cd}$ as a function of the time-of-flight (ToF). In this way the quality of the nuclear data for these nuclides and the optimal thickness of the SINRD filters obtained in [6] were verified.

During the SINRD benchmark experiments an automatic sample changer was positioned at $7.7 \mathrm{~m}$ from the neutron producing target, allowing an automated alternation of sample-in $\left(\varphi_{t r}\right)$ and sample-out $\left(\varphi_{0}\right)$ measurements. A second sample changer, which was placed close to the sample position and is not included in Fig. 1, was used for background filters. Background filters

(C) The Authors, published by EDP Sciences. This is an Open Access article distributed under the terms of the Creative Commons Attribution License 4.0 (http://creativecommons.org/licenses/by/4.0/). 




Figure 1. Schematic view of a transmission experiment.

Table 1. Characteristics of the $\mathrm{Gd}$ and $\mathrm{Cd}$ samples used for the transmission measurements. All samples were in the form of a metal foil or disc.

\begin{tabular}{lcc} 
Element & $\begin{array}{c}\text { Nominal } \\
\text { thickness }(\mathrm{mm})\end{array}$ & $\begin{array}{c}\text { Areal density } \\
\left(10^{-3} \mathrm{at} / \mathrm{b}\right)\end{array}$ \\
\hline $\mathrm{Gd}$ & 0.030 & $0.0835 \pm 0.0001$ \\
$\mathrm{Gd}$ & 0.100 & $0.3200 \pm 0.0002$ \\
$\mathrm{Gd}$ & 0.200 & $0.6287 \pm 0.0001$ \\
$\mathrm{Cd}$ & 0.500 & $2.3598 \pm 0.0001$ \\
$\mathrm{Cd}$ & 1.000 & $4.6668 \pm 0.0004$ \\
$\mathrm{Cd}$ & 1.000 & $4.6765 \pm 0.0007$ \\
\hline
\end{tabular}

are used to estimate the neutron background during the experiments according to the black resonance technique [12].

Neutrons traversing the sample and the filters are finally detected by a $6.35 \mathrm{~mm} \times 76 \mathrm{~mm} \times 76 \mathrm{~mm}$ Li glass scintillator, placed at $11 \mathrm{~m}$ from the neutron producing target. The detector, which is enriched to $95 \%$ in ${ }^{6} \mathrm{Li}$, is directly connected to a photomultiplier tube. A good transmission geometry is realized by proper collimation of the neutron beam. A set of $\mathrm{Li}, \mathrm{B}, \mathrm{Cu}, \mathrm{Ni}$ and $\mathrm{Pb}$ collimators with decreasing diameter are placed between the neutron producing target and the sample; a similar sequence of collimators with increasing diameter are placed between the sample and the detector. Experiments in good transmission geometry are required to ensure that all detected neutrons traverse the sample and scattered neutrons do not reach the detector [12].

Transmission measurements using $\mathrm{Gd}$ and $\mathrm{Cd}$ metal foils or discs of different thicknesses were carried out. The characteristics of the samples are summarized in Table 1. The beam size at the sample position had a 10-mm diameter. To reduce the influence of the $\gamma$-ray flash in the detector, a permanent $\mathrm{Pb}$ filter was installed in the beam line close to the neutron producing target.

The experimental transmission $\left(\mathrm{T}_{\text {exp }}\right)$ is defined as the ratio between sample-in $\left(\varphi_{t r}\right)$ and sample-out $\left(\varphi_{0}\right)$ measurements and was calculated according to Formula (2). The experimental value of the transmission was computed as the ratio between the ToF spectra obtained with the sample in the beam $\left(\mathrm{C}_{\mathrm{tr}}\right)$ and the unperturbed case $\left(\mathrm{C}_{0}\right)$. Both contributions were corrected for the corresponding background component, $\mathrm{B}_{t r}$ and $\mathrm{B}_{0}$ respectively.

$$
T_{\exp }(E)=\frac{\varphi_{t r}(E)}{\varphi_{0}(E)}=\frac{C_{t r}(E)-B_{t r}(E)}{C_{0}(E)-B_{0}(E)}
$$

The ToF spectra were corrected for losses due to the dead time in the detector and electronics chain, and all spectra were normalized to the same neutron intensity and ToF-bin width. The background, which is a sum of ToF independent and dependent components, was determined

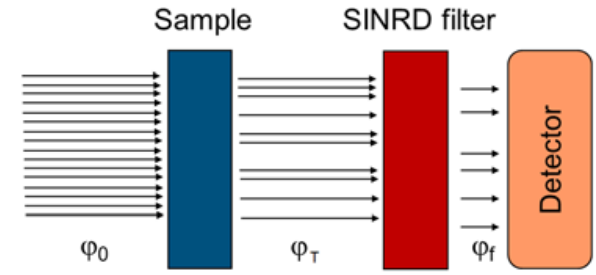

Figure 2. Schematic representation of the self-indication experiments carried out at GELINA.

by applying the black resonance technique [12]. The resonance dips resulting from $\mathrm{Co}$ and $\mathrm{Na}$ filters placed permanently in the beam were used to account for the impact of the presence of the sample on the background. The average background $\left(\mathrm{B}_{\mathrm{tr}}, \mathrm{B}_{0}\right)$ below $1 \mathrm{eV}$ was about $4 \%$ of the sample-in and sample-out measurements $\left(\mathrm{C}_{\mathrm{tr}}, \mathrm{C}_{0}\right)$.

The experimental transmission $\left(\mathrm{T}_{\exp }\right)$ was compared to the analytical estimate $\left(\mathrm{T}_{\mathrm{an}}\right)$. The latter was calculated with Formula (3), where $\mathrm{n}_{\text {fil }}$ is the areal density and $\sigma_{\text {fil }}$ is the total cross-section of the SINRD filter. The cross-section values were obtained from the JANIS software [13] with the ENDF/B-VII.0 nuclear data library [14].

$$
T_{a n}(E)=\exp \left[-n_{f i l} \sigma_{f i l}(E)\right] .
$$

\subsection{Benchmark measurements}

Validation experiments were carried out to demonstrate the basic principle of the SINRD techniques and to compare the response of different detector types. The setup of the experiments is reported in Fig. 2.

The incoming neutron flux $\left(\varphi_{0}\right)$ interacts with a sample under investigation and with the so-called SINRD filter to obtain the transmitted flux $\left(\varphi_{\mathrm{T}}\right)$ and the filtered flux $\left(\varphi_{\mathrm{f}}\right)$, respectively. The sensitivity to the sample is enhanced by combining measurements with different SINRD filters and a detector sensitive to a specific resonance of a material of interest in the sample.

Due to safety regulations and limitations of available material, measurements with natural $\mathrm{Cd}$-samples with different thicknesses were used to mimic the presence of ${ }^{239} \mathrm{Pu}$ in spent fuel. The neutron cross section of ${ }^{113} \mathrm{Cd}$ has a strong resonance at $0.167 \mathrm{eV}$, which is relatively close in energy to the $0.296 \mathrm{eV}$ resonance of ${ }^{239} \mathrm{Pu}$. The areal density of the $\mathrm{Cd}$ sample that is placed in the beam can be derived from the attenuation of the neutron beam due to the $0.167 \mathrm{eV}$ resonance.

To construct a detector with a high sensitivity to the $0.167 \mathrm{eV}$ energy region, a thin Cd sample ( $0.027 \mathrm{~mm}$ thick) was surrounded by $4 \mathrm{C}_{6} \mathrm{D}_{6}$ liquid scintillators detecting the prompt $\gamma$-rays emitted after $(\mathrm{n}, \gamma)$ reaction. This detection system was placed at a $13.2 \mathrm{~m}$ distance from the neutron producing target. Measurements with a Gd or Cd SINRD filter in the beam were performed to measure neutrons with energy higher than the cut-off energy of each filter. By calculating the difference between the two measurements, the neutron flux close to $0.167 \mathrm{eV}$ was estimated. The thickness of these filters was optimized for the detection of neutrons with energy close to $0.167 \mathrm{eV}$, therefore slight changes are anticipated from the values proposed for ${ }^{239} \mathrm{Pu}$ measurements. The characteristics of the SINRD filters are reported in Table 1. The SINRD filters were placed 
Table 2. Characteristics of the Cd samples used for the validation measurements. All samples were in the form of a metal disc of $80 \mathrm{~mm}$ diameter.

\begin{tabular}{ccc} 
Element & $\begin{array}{c}\text { Nominal } \\
\text { thickness }(\mathrm{mm})\end{array}$ & $\begin{array}{c}\text { Areal density } \\
\left(\mathrm{x} \mathrm{11} 0^{-4} \mathrm{at} / \mathrm{b}\right)\end{array}$ \\
\hline $\mathrm{Cd}$ & 0.030 & $1.3611 \pm 0.0001$ \\
$\mathrm{Cd}$ & 0.050 & $2.2283 \pm 0.0001$ \\
$\mathrm{Cd}$ & 0.075 & $3.3845 \pm 0.0001$ \\
\hline
\end{tabular}



Figure 3. Transmission through different $\mathrm{Gd}$ and $\mathrm{Cd}$ foils. The experimental transmission is compared with the analytical transmission.

far from the detectors to avoid background contribution of neutron capture reactions in the filters. Measurements were performed using cadmium samples with different thicknesses, and the samples were placed at about $6.5 \mathrm{~m}$ from the neutron producing target. The characteristics of these samples are summarized in Table 2.

The results obtained with the self-indication geometry were compared with measurements with Frisch gridded ionization chambers with thin deposits of ${ }^{235} \mathrm{U}$ or ${ }^{10} \mathrm{~B}$. These detectors do not show an enhanced efficiency at the energy of the resonance of interest, i.e. the $0.167 \mathrm{eV}$ resonance. The detectors were positioned at a $7.65 \mathrm{~m}$ distance from the neutron production target.

\section{Results}

\subsection{Transmission experiments}

The results from the experimental transmission are shown in Fig. 3 for the different $\mathrm{Gd}$ and $\mathrm{Cd}$ filters. The plot includes also the values obtained with the analytical approach of Formula (3).

The analytical calculations do not include the response of the ToF-spectrometer; however, in the low energy region, the influence of this response is small compared to the broadening of the resonance profiles due to the Doppler effect and the total width of the resonance.

The spectra in Fig. 3 illustrate that all Gd foils have a cut-off energy below the $\mathrm{Cd}$ resonance at $0.167 \mathrm{eV}$, whereas the cut-off energy for $\mathrm{Cd}$ is slightly above $0.3 \mathrm{eV}$. Evidently the cut-off energy increases with increasing filter thickness. In order to reduce the contribution from thermal neutrons, visible in Fig. 3 in the case of $0.5 \mathrm{~mm}$ thick $\mathrm{Cd}$ filter, a $\mathrm{Cd}$ filter with a minimum thickness of $1.0 \mathrm{~mm}$ is suggested. The results in Fig. 3 show an overall



Figure 4. Experimental observable $\mathrm{R}_{\mathrm{SI} \text {,exp }}$ as a function of the areal density of the $\mathrm{Cd}$ sample placed in the beam.

good agreement between the experimental and analytical transmissions.

From the results shown in Fig. 3, a thickness of about $0.10 \mathrm{~mm}$ for the Gd filter is suggested to obtain a cut-off energy slightly below the resonance of ${ }^{239} \mathrm{Pu}$ at $0.296 \mathrm{eV}$. As mentioned above, a thickness of $1.0 \mathrm{~mm}$ for the $\mathrm{Cd}$ filter is recommended to have a cut-off above the same resonance energy and to reduce the contribution from neutrons below $0.1 \mathrm{eV}$. Therefore, the combinations of SINRD filters identified in [8] to derive the amount of ${ }^{239} \mathrm{Pu}$ are confirmed with these experimental results.

Due to the lower energy of the Cd resonance compared to the one of ${ }^{239} \mathrm{Pu}$, the filter thickness of the Gd filter has to be adapted for the benchmark measurement at GELINA. Following the criteria described above, the optimum filter thicknesses are $0.03 \mathrm{~mm}$ for the Gd filter and $1.0 \mathrm{~mm}$ for the $\mathrm{Cd}$ filter in case of self-indication measurements with $\mathrm{Cd}$ as material of interest.

\subsection{Benchmark experiments}

Self-indication experiments using a neutron detector consisting of a $0.027 \mathrm{~mm}$ thin Cd sample combined with $4 \mathrm{C}_{6} \mathrm{D}_{6}$ detectors were carried out with SINRD filters in the beam. Measurements were performed with $0.03 \mathrm{~mm}$, $0.05 \mathrm{~mm}, 0.075 \mathrm{~mm}$ and $0.105 \mathrm{~mm} \mathrm{Cd}$ samples placed in the neutron beam.

The obtained ToF spectra were normalized to the same neutron intensity using the total counts of a $\mathrm{BF}_{3}$ proportional counter which is installed in the concrete ceiling of the GELINA target hall.

An observable was defined for the measurements with the SINRD filters, by taking the difference between the total counts resulting from the measurements with the $\mathrm{Gd}$ and $\mathrm{Cd}$ filters in the beam. The $\mathrm{R}_{\mathrm{SI} \text { exp }}$ signature is defined in Formula (4), and $\mathrm{C}_{G d}$ and $\mathrm{C}_{C d}$ are the total counts of the spectra taken with the $\mathrm{Gd}$ and $\mathrm{Cd}$ filters, respectively. The neutron background was subtracted for both spectra.

$$
R_{S I, \exp }=\frac{1}{C_{G d}-C_{C d}}
$$

The results obtained with the self-indication detector are shown in Fig. 4 with the ones obtained using a ${ }^{235} \mathrm{U}$ fission chamber and a ${ }^{10} \mathrm{~B}$ ionisation chamber as neutron detectors. It was supposed that the neutron background 
was constant during the measurements with the ${ }^{235} \mathrm{U}$ and ${ }^{10} \mathrm{~B}$ detectors, therefore it cancels out in the calculation of the observable. The values in Fig. 4 have been normalized to have $R_{\text {SI, exp }}=1$ for the measurements without Cd sample.

The highest sensitivity to the $\mathrm{Cd}$ areal density is obtained with the self-indication detector and this confirms the advantage of using the self-indication technique. The large SINRD signature for the self-indication detector is due to the high detection efficiency to neutron with energy close to the resonance of interest (i.e. $0.167 \mathrm{eV}$ ). The results calculated for the ${ }^{235} \mathrm{U}$ fission chamber are more sensitive to the $\mathrm{Cd}$ areal density compared to the values obtained for the ${ }^{10} \mathrm{~B}$ ionization chamber. This is due to the resonance of the ${ }^{235} \mathrm{U}$ total cross-section at $0.271 \mathrm{eV}$, which is close to the resonance energy of $\mathrm{Cd}$.

The results in Fig. 4 demonstrate that the optimal results are achieved by using a detector with enhanced detection efficiency close to a resonance of the material of interest. Hence, to determine the amount of ${ }^{239} \mathrm{Pu}$ in a spent fuel assembly a ${ }^{239} \mathrm{Pu}$ based fission chamber is recommended. This result is in line with the conclusion in [8], where the SINRD signature calculated with the ${ }^{239} \mathrm{Pu}$ fission chamber showed the highest sensitivity to the ${ }^{239} \mathrm{Pu}$ content in the fuel compared to other detectors.

\section{Conclusion}

The results of the SINRD benchmark experiments performed at the GELINA facility of JRC-Geel were summarized in this contribution.

Transmission measurements were performed to verify the quality of nuclear data used in the previous Monte Carlo simulation for the optimization of the SINRD filters. The comparison showed that the quality of nuclear data is sufficient to define the optimal thickness of the $\mathrm{Gd}$ and $\mathrm{Cd}$ filters. The results of the experiments indicated that the combination of a Gd filter of about $0.1 \mathrm{~mm}$ and a $1.0 \mathrm{~mm}$ $\mathrm{Cd}$ filter is suggested for the measurement of spent fuel containing ${ }^{239} \mathrm{Pu}$.
In addition, the results from self-indication experiments were compared with the values obtained for a ${ }^{235} \mathrm{U}$ fission chamber and a ${ }^{10} \mathrm{~B}$ ionization chamber, and the results confirmed that the highest sensitivity is obtained using a neutron detector with an enhanced efficiency for a resonance of the material of interest. Therefore, a ${ }^{239} \mathrm{Pu}$ fission chamber is recommended for the characterisation of spent fuel by SINRD.

The research is sponsored by Engie in the framework of the cooperation agreement CO-90-07-2124 between SCK •CEN and Engie. The authors acknowledge the support of the EURATOM Fission 7th Framework Programme's project GENTLE (grant number 323304) for the experiments at the GELINA facility.

\section{References}

[1] Menlove H.O., et al., Nucl. Appl. 6, 401-408 (1969)

[2] LaFleur A. M., Ph.D. diss. at TA\&MU (2011)

[3] Hu J., et al., JNMM XL-3 (2012)

[4] LaFleur A.M., et al., Nucl. Tec. 181, 354-370 (2013)

[5] LaFleur A.M., et al., Nucl. Instr. and Meth. Sec. A 781, 86-95 (2015)

[6] Rossa R., et al., Proc. 55th INMM meeting (2014)

[7] Rossa R., et al., Ann. Nucl. En. 75, 176-183 (2015)

[8] Rossa R., et al., Nucl. Instr. and Meth. Sec. A 791 93-100 (2015)

[9] Fröhner F. H., et al., Conf. on Neutr. Cross Section Tech., CONF-660 303 1, 55-66 (1966)

[10] Massimi C., et al., J. Kor. Ph. Soc. 59, 1689-1692 (2011)

[11] Schillebeeckx P., et al., JRC Science and Policy Reports - Report EUR 26848 EN (2014)

[12] Schillebeeckx P., et al., Nucl. Data Sheets 113, 3054-3100 (2012)

[13] Soppera N., et al., Nuc. Data Sheets 120, 294-296 (2014)

[14] Chadwick M.B., et al., Nuc. Data Sheets 107, 12 (2006) 